\title{
Research on Service-oriented Manufacturing Supply Chain*
}

\author{
Jianbiao Chen \\ Guangzhou College of Technology and Business \\ Guangzhou, China 510800
}

\author{
Ying Liu \\ Guangzhou College of Technology and Business \\ Guangzhou, China 510800
}

\begin{abstract}
This paper takes the service-oriented manufacturing supply chain as the research object. Through the study of a large number of related literatures of the service-oriented manufacturing supply chain, it is found that the research results related to the evolution path of the serviceoriented manufacturing supply chain are very few. And the evolution path of the service-oriented manufacturing supply chain is the key problem to be solved in the process of manufacturing transformation. The existing research results are sorted out and summarized from three aspects: serviceoriented manufacturing industry, supply chain network evolution and service-oriented manufacturing supply chain. Finally, four key links are proposed to study the evolution path of service-oriented manufacturing supply chain.
\end{abstract}

Keywords—service-oriented manufacturing; supply chain; evolutionary path

\section{INTRODUCTION}

Efficient service-oriented manufacturing supply chain network is the main embodiment of future core competitiveness. Enterprises strive to put themselves in an efficient supply chain network of service-oriented manufacturing industry, which is one of the most important ways to maintain the sustainable development of enterprises and always have competitive advantages. At present, there are few studies on the supply chain network of serviceoriented manufacturing industry, most of which only focus on the mode of transformation from traditional manufacturing industry to service-oriented manufacturing industry. The research contents of service-oriented manufacturing industry mainly focus on two aspects. One is manufacturing-oriented service, which is mainly reflected on the extension of traditional manufacturing industry in business, from producing tangible products to "tangible products + services", such as Honeywell Company, Shaanxi Blower Group Co. Ltd, etc. The other is service-oriented manufacturing, which is mainly reflected on the penetration of traditional service industry into manufacturing industry, from single service to "service + tangible products", such as, China Mobile.

However, today's enterprises are no longer isolated

*Fund: The subject co-construction project of 13th Five-Year Plan of Philosophy and Social Sciences of Guangdong Province in 2017 "Innovation Model of Service-oriented Manufacturing Industry in Guangdong-Hong Kong-Macao Greater Bay Area" (No. GD17XTQ01). entities. As the division of labor becomes more and more detailed, enterprises must put themselves in a consortium if they want to survive, which is called "supply chain network". The supply chain network of service-oriented manufacturing industry is quite different from the supply chain network of manufacturing industry and the supply chain network of service industry studied by most scholars at present in connotation and structure. Customers and experiences of service affect the design and transmission of "product service system", which is the remarkable feature of service-oriented manufacturing. Moreover, customers' time cost, physical cost, psychological cost and the cost of using products ultimately determines the composition of the comprehensive value of service-oriented manufacturing [7]. From this point of view, it is wrong to equate the supply chain network of manufacturing industry and the supply chain network of service industry with the supply chain network of serviceoriented manufacturing industry. Therefore, only by combining the service-oriented manufacturing industry with the supply chain network and constructing the supply chain network of the service-oriented manufacturing industry, can enterprises have the opportunity to maintain their competitive advantage in the fierce competitive environment and obtain the power of sustainable development.

At present, there are few studies on the supply chain network of service-oriented manufacturing industry in China and foreign countries, most of which are limited to the research of service-oriented manufacturing industry or the evolution of supply chain network. Combining the two, there are few related research results on supply chain network of service-oriented manufacturing industry. Some scholars have studied the hybrid supply chain of service-oriented manufacturing industry, but not the evolution path of supply chain network of service-oriented manufacturing industry.

This paper combs the existing research results from three aspects: service-oriented manufacturing industry, supply chain network evolution and service-oriented manufacturing supply chain, and puts forward that the future research direction should focus on the evolution path of serviceoriented manufacturing supply chain network.

\section{RESEARCH ON SERVICE-ORIENTED MANUFACTURING INDUSTRY}

Service-oriented manufacturing is the product of the gradual integration of manufacturing industry and service 
industry in the process of independent development. Serviceoriented manufacturing can be divided into manufacturingoriented services and service-oriented manufacturing. At present, many scholars have studied service-oriented manufacturing.

Timothy D. Fry, Daniel C. Steele, Brooke A. Saladin, et al. (1994) first put forward service-oriented manufacturing strategy, and introduced the concept of service-oriented manufacturing strategy [1].

Fujimoto Jun, Umeda Yasushi, Tamura Tetsuya, Tomiyama Tetsuo, Kimura Fumihiko et al. (2004) studied the service-oriented products developed based on reverse manufacturing concept. The results showed that serviceoriented business could potentially reduce the impact on the environment and expand business opportunities from the perspective of the whole product life cycle [2].

Sun Linyan, Li Gang, Jiang Zhibin, Zheng Li, He Zhe, etc. (2007) first put forward the advanced manufacturing mode, namely, service-oriented manufacturing, which adapts to the trend of integration of manufacturing and service in China. The new problems to be solved urgently in the research of service-oriented manufacturing are highlighted [3].

Rubén Darío Franco, Ángel Ortiz Bas, Francisco Lario Esteban, et al. (2009) studied the modeling of extended manufacturing process by service-oriented entities [4]. Jiaqi Yan, Kang Ye, Huaiqing Wang, Zhongsheng Hua, etc. adopted strategic consistency model to study service-oriented collaborative manufacturing [5]. Xi Zhuyan, Zheng Bo and Sun Linyan (2009) studied the reasons why the manufacturing industry in Shandong Province is not strong and the service industry lags behind from the perspective of service-oriented manufacturing strategy. From the two aspects of the proportion of service in the product service system and the organizational structure, they put forward a path model for manufacturing enterprises to transform from service-oriented manufacturing strategy to service-oriented manufacturer. Lin Wenjin, Jiang Zhibin, Li Na and others (2009) sorted out the research results of service-oriented manufacturing theory, put forward that the theoretical basis of service-oriented manufacturing was traditional manufacturing theory, behavioral science, organization theory, social network theory and complex system theory, conceived the theoretical system of service-oriented manufacturing, and put forward some key issues to be studied at this stage [7].

Hasse Nylund, Paul H. Andersson et al. (2010) studied service-oriented and distributed manufacturing system modeling and simulation [8]. Paulo Leitão, Joel Alves, Ana I. Pereira, etc. (2010) used Petri net model to study real-time decision-making in service-oriented manufacturing systems [9]. Wang Mingwei, Zhang Shusheng, Zhou Jingtao, etc. (2010) studied the framework of collaborative business process building for service-oriented manufacturing, and studied three key technologies of manufacturing service semantic modeling, service discovery matching and service composition within the framework [10].
Shuangxi Huang, Sen Zeng, Yushun Fan, George Q. Huang and others (2011) studied the optimal service selection and composition of service-oriented manufacturing networks. A method based on uncertainty and genetic algorithm was proposed to achieve the optimal service selection and composition in an effective and efficient manner [11]. Jie Gao, Yinliang Yao, Valerie C. Y. Zhu, Linyan Sun, Lin Li and others (2011) studied the characteristics of the three product and service systems and their transformation [12]. Cheng Gongsheng (2011) studied the problems faced by the development of service-oriented manufacturing in Guangdong Province and put forward countermeasures and suggestions [13]. Chen Juhong, Jiao Xingjia, Guo Fuli, etc. (2011) studied the design of product service system in service-oriented manufacturing from the aspects of design objectives, conceptual design and detailed design stage content, organizational form and knowledge dependence. Cui Jiachen, Lin Wenjin, Wang Shuai and Jiang Zhibin (2011) studied the mechanism of customer value transmission under service-oriented manufacturing mode. The results show that customer participation has a positive impact on service capability, and service capability has a positive impact on perceived value and customer satisfaction [15].

Corina Popescu, M. Cavia Soto, Jose L. Martinez Lastra (2012) studied the method of incremental modeling of traffic and resources in service-oriented manufacturing systems based on Petri nets [16]. H. Guo, F. Tao, L. Zhang, Y. J. Laili, D. K. Liu and others have studied the measurement method of flexibility of resource service composition in service-oriented manufacturing systems [17]. Fei Tao, Ying Cheng, Lin Zhang, Dongming Zhao (2012) studied the comprehensive utility model of the resource service transaction chain of "multi-resource service provider — one agent - multi-resource service demander" under centralized decision-making conditions. The results show that there is a utility equilibrium between the resource service provider and the agent [18]. Fuqiang Zhang, Pingyu Jiang, Qiqi Zhu, Wei Cao (2012) applied integrated analytic hierarchy process and complex network theory to study the cluster characteristics of service-oriented enterprise collaboration network [19]. Lu Zhen (2012) studied the service-oriented manufacturing strategy and proposed an optimal decision model for serviceoriented manufacturing [20]. Cheng Dongquan, Gu Feng and Geng Yong (2012) studied the structure and operation mechanism of value chain system in service-oriented manufacturing, and proposed that the value chain creation model can be summarized as "three-all" mode [21]. Yao Qifu, Xiong Huiping and Wang Hongjun (2012) studied the implementation path of service-oriented manufacturing based on Ningbo manufacturing industry [22]. Xie Wenming, Jiang Zhibin, Chu Yibing et al. (2012) studied the application of service-oriented manufacturing in Shanghai Electric, and summarized two modes of service-oriented manufacturing in Shanghai Electric: symbiotic service mode and endogenous service mode [23].

Cristina Morariu, Octavian Morariu, Theodor Borangiu, Yves Sallez (2013) studied a service-oriented manufacturing system for intelligent products [24]. Yang Lixi and Tang 
Zhenpeng (2013) studied the development thinking and countermeasures of service-oriented manufacturing mode in Haixi Economic Zone, and put forward the overall development thinking of service-oriented manufacturing mode in Haixi Economic Zone [25].

Kangzhou Wang, Zhibin Jiang, $\mathrm{Na} \mathrm{Li}$ and $\mathrm{Na}$ Geng (2014) studied the optimal production control of serviceoriented manufacturing systems based on customer tracking behavior [26]. Based on the data of 200 manufacturing enterprises in Jiangsu Province, Yang Hui, Song Huaming and Yu Anping (2014) analyzed the competitive advantages of service-oriented manufacturing mode. The results showed that the main components of service-oriented manufacturing mode had positive effects on enterprise performance and customer performance respectively [27].

Bai Li Guo (2015) studied the optimization theory of supply chain based on service-oriented manufacturing, and analyzed the relationship between service-oriented manufacturing and hybrid supply chain [28]. Zhang Zhong and Jin Qing (2015) studied the value creation process of manufacturing enterprises based on service-oriented manufacturing network, and put forward four ways [29] for manufacturing enterprises to participate in the value creation of service-oriented manufacturing network, such as knowledge-driven, modular innovation of service-oriented production, outsourcing of productive services and green value creation.

Jin Cao, Zhibin Jiang, Kangzhou Wang (2016) studied customer demand forecasting for service-oriented manufacturing based on customer satisfaction [30]. Liangqing Feng, Kunkun Zeng, Fangling Shu (2016) studied the problem of reputation quality control in manufacturing networks based on incentive services, and proposed an optimal dynamic model of quality behavior in manufacturing dynamic network nodes [31]. Liu Zhiyong, Li Bizhen, Ye Songzhong, Yang Shaoxiong and others (2016) studied the different paths of service-oriented manufacturing development of Fujian sports goods manufacturing enterprises [32].

Ying Cheng, Fei Tao, Dongming Zhao, Lin Zhang (2017) studied the modeling of service supply-demand matching hypernetwork in service-oriented manufacturing systems [33]. Cao Yuping (2017) took Weifang City of Shandong Province as the research background, and studied the path of developing service-oriented manufacturing in the area of textile industry transformation [71]. Based on the contingency theory of environment - strategy - structure, Jian Zhaoquan, Liu Xiaoyan and Li Lei (2017) studied the organization structure of "platform + small and micro enterprise" of Haier's service-oriented manufacturing enterprises.

Julia Siderska, Khair S. Jadaan (2018) studied serviceoriented manufacturing in cloud manufacturing [35]. Yan Kaining and Li Gang (2018) studied the transformation of service-oriented manufacturing enterprises against the background of "Internet", and put forward the evolution path of enterprise change oriented by intellectualization, platform and networking [36]
All of the above are based on the research results of the individual enterprise transforming to the enterprise of service-oriented manufacturing. They didn't put the enterprise in a complete product supply system to make the research, lacking the systematicness. In today's competitive environment, the competitiveness of enterprises should not only be evaluated by themselves, but also by the supply chain in which they are located. Therefore, the first step of enterprise transformation is to study the individual enterprise transforming to the enterprise of service-oriented manufacturing. If an enterprise wants to win, it must study how to put itself in an efficient service-oriented manufacturing supply chain network. Enterprises must change their thinking and devote themselves to the construction of service-oriented manufacturing supply chain network in the process of transforming to service-oriented manufacturing, so as to achieve real competitive advantage.

\section{RESEARCH ON THE EVOLUTION OF SUPPLY CHAIN NETWORK}

There have been many studies on supply chain evolution both in China and foreign countries.

David Sadler (2004) studied the decline of the iron and steel industry in northeastern England and analyzed the importance of dynamic cluster evolution [37]. Li Jizi, Cai Gennv and Lu Yinde (2004) studied the evolution of supply chain based on cluster network [38]. Wang Yongping and Meng Weidong (2004) used evolutionary game theory to establish a mathematical model of the evolutionary game of the cooperative competition mechanism of supply chain enterprises, and analyzed the dynamic process of the evolution of the cooperative competition mechanism of supply chain enterprises [39].

Masayoshi Maruyama, Mitsunori Hirogaki (2007) studied the evolution of the supply chain of fresh agricultural products, and proposed a three-level supply chain model composed of producers, wholesalers and retailers. The model was affected by two uncertainties of product quality and retail demands [40]. Wang Ling (2007) used the single population game and dual population game of evolutionary game theory to analyze the evolution process of horizontal and vertical competition of supply chain network and the influencing factors of evolutionary equilibrium [41].

Tim Coltman, Kathrine Bru, Nidthida PermAjchariyawong, Timothy M. Devinney, Gabriel R.G. Benito (2008) studies the evolution of supply chain contract based on the theory of organization and new system [42].

V. Daniel R. Guide, Luk N. Van Wassenhove (2009) studied the evolution of closed-loop supply chain [43].

Wang Shilei and Yan Guangle (2009) used evolutionary game theory to build an evolutionary game model between suppliers and core enterprises. The analysis results of the model showed that the evolution of the system presented the characteristics of "path dependence" [44]. 
Ozcan Ozan, Reeves Kingsley, Ozcan Deniz (2010) studied the evolution process of supply chain structure of companies focusing on sustainable development [45].

Chai Guorong, Li Zhenchao, Wang Xiaogeng and Zong Shengliang (2011) studied the evolution of cooperative behavior of cluster enterprises under supply chain network. The results showed that the evolution of cooperative behavior of cluster enterprises depended on the initial state of cooperation, return on assets, income adjustment and network income [46].

Zhou He, Shouyang Wang, T.C.E. Cheng (2013) studied the competition and evolution process in multi-product supply chain based on agent retailer model. The results showed that the ability of consumers to collect pricing information had a significant impact on the competitiveness of retail chains [47].

From the perspective of ecological niche, innovation strategy and ecological niche expansion, Ji Guojun, Gunasekaran, Angappa (2014) studied the impact of supply chain cluster growth and the matching degree of the impact of supply chain cluster growth, and introduced the ecological niche expansion model, growth model and growth evolution model of supply chain cluster [48]. Wu Yisheng (2014) studied the evolution model of collaborative operation of low-carbon supply chain, and analyzed the evolution process of order parameters affecting the collaborative operation of low-carbon supply chain by using self-organization principle [49].

Xue Xiao, Wang Shufang, Chao Hao (2015) studied the autonomous evolution of service system in cluster supply chain, and proposed a feedback-based evolution mechanism of service system in cluster supply chain [50]. Zhang Guoxing, Fang Shuai and Wang Yingluo (2015) studied the coordination mechanism of supply chain based on evolutionary game. The results showed that the smaller the cost of coordination supply chain and the larger the overall excess revenue obtained will help the whole system evolve toward the direction of cooperation [51].

Ying Xu, Xuemei Zhang, Jian Cao, Ya Chen, Xuhong Ye, Paolo Renna (2016) studied the supply chain of e-commerce and express industry, and analyzed the cooperative operation of enterprises [52].

Wang Ying and Zhang Chun (2017) studied the governance model pf supply chain and its evolution, and summarized several typical evolution paths of supply chain governance model, such as, from stochastic transaction to core leadership, from core leadership to multi-collaboration, and from core leadership to rotary stochastic transaction [53].

Based on the complexity of supply chain network, Hua Wang, Tao Gu, Maozhu Jin, Rong Zhao, Guanxiang Wang (2018) studied the complexity of supply chain network structure in the context of interruption risk, and proposed a method for measuring the complexity and evolution of ER stochastic network, small world network and BA scale-free network [54]. Tang Run and Peng Yangyang (2018) studied the evolutionary game of dual-channel coordination in fresh food supply chain considering channel characteristics. The payment matrix of dual-population evolution in dual-channel supply chain was characterized by the optimal function of differential game, and the evolutionary stability was judged and the evolutionary path of the system was analyzed [55]. He Binbin and Liu Qin (2018) applied evolutionary game theory to study the vertical cooperative evolutionary game of supply chain with consumers' green preferences. The results showed that consumers' green preferences had an important impact on the marketing model of supply chain [56].

The above research results mainly focus on the evolution process of supply chain in traditional manufacturing industry, not on the evolution of supply chain network in serviceoriented manufacturing industry. The service-oriented manufacturing supply chain is the deep integration of manufacturing supply chain and service supply chain. It is a more complex system. The evolution mechanism of serviceoriented manufacturing supply chain can't be simply analyzed by the evolution process of manufacturing supply chain or service supply chain. In order to understand the evolution mechanism of service-oriented manufacturing supply chain fundamentally, enterprises must re-analyze the characteristics of manufacturing and service industries after deep integration.

\section{SERVICE-ORIENTED MANUfACTURING SuPPly CHaIN}

At present, the research on the supply chain of serviceoriented manufacturing industry started relatively late, and the existing research results are not much. The following is a summary of the main research results of the supply chain of service-oriented manufacturing industry.

Yunus Kathawala, Khaled Abdou (2003) evaluated the difference between the supply chain of service industry and the supply chain of manufacturing industry. The results showed that an important factor to improve efficiency and effectiveness is to strike a balance between reducing costs and improving service quality [57].

Kaushik Sengupta, Daniel R. Heiser, Lori S. Cook (2006) made a comparative analysis of supply chain performance in manufacturing and service industries, which showed that before implementing a specific supply chain strategy, the practicing manager needed to determine the appropriate benchmark and competitive priority [58].

Wang Chunzhi, Heiko Gebauer, Sun Liyang (2008) studied the construction of service management system of manufacturing enterprises based on supply chain management, and analyzed the strategic framework of service management of manufacturing enterprises from upstream to downstream with manufacturing enterprises as the main management body [59].

He Zhe, Sun Linyan, He Zhuxin and Li Gang (2008) studied the rise of service-oriented manufacturing and its differences between the supply chain of service-oriented manufacturing with the traditional supply chain system, put forward the concept and attributes of service-oriented manufacturing, and differentiated it from the traditional logistics supply chain system in terms of profit model, 
organizational behavior, circulation system, value appeal and drawing mechanism, network circulation carrier, etc. [60].

Wang Ji-Peng, Xiong Jing, and Wang Tian-Lin (2011) studied the implementation of node interface in manufacturing agile supply chain based on service agent. The results showed that the use of autonomous, adaptive and collaborative service agents to achieve supply chain agility and reconfigurability can support supply chain enterprises of manufacturing industry to restructure and adjust the supply chain rapidly and intelligently at low cost [61].

Tang Yan, Li Jian, Zhang Jihui (2012) studied the design of cloud manufacturing service platform of closed-loop supply chain for remanufacturing. Through resource integration, demand docking and service integration, the cloud manufacturing service platform of closed-loop supply chain for remanufacturing was constructed [62].

Wang Kangzhou, Jiang Zhibin, Lin Wenjin and Xie Mingming (2013) studied the management of hybrid supply chain for service-oriented manufacturing, analyzed the bullwhip effect of hybrid supply chain, the main problems of production and service capability management, and the impact of service on supplier relationship management and performance evaluation in supply chain [63]. Ma Chao, Wang Jun, Gao Song (2013) studied the evaluation of collaboration capability of hybrid supply chain in serviceoriented manufacturing, and proposed a maturity model of collaboration capability of hybrid supply chain [64].

Jukka Hemilä, Jyri Vilko (2015) studied the construction of service supply chain model for small and medium-sized manufacturing enterprises, and proposed that the new knowledge needed for service business development in supply chain environment [65].

James Aitken, Paul Childerhouse, Eric Deakins, Denis Towill (2016) made a comparative analysis of supply chain integration between manufacturing industry and service industry based on uncertain cycle model [66]. Ray Y. Zhong, Stephen T. Newman, George Q. Huang, Shulin Lan (2016) studied the challenges, opportunities and trends of supply chain management in service-oriented manufacturing industry against the background of big data. Dan Bin, Luo Xiao, Liu Molin (2016) studied the supply chain model of product service integrated in manufacturing and service process, and proposed four typical models of product service supply chain based on after-sales integration, sales integration, production integration and design integration [68].

Wang Weiwei, Hong Yue and Zhang Zaifang (2017) studied the stability and elasticity evaluation of hybrid supply chain for service-oriented manufacturing, and proposed a analysis model of automatic feedback compensation system for production services of hybrid supply chain [69].

Ehsan Pourjavad, Arash Shahin (2018) applied fuzzy theory to evaluate the performance of sustainable service and supply chain management of manufacturing industry. The results showed that sustainable manufacturing standards had a greater impact on the performance of sustainable supply chain management than sustainable service standards [70].

In summary, the research on service-oriented manufacturing supply chain is basically confined to structure, coordination mode and performance evaluation, lacking of research on the evolution of supply chain network in serviceoriented manufacturing. The evolution path of serviceoriented manufacturing supply chain is the guiding light for enterprises to transform, and plays a key role in the successful transformation of enterprises. The environment of enterprises has great influence on the evolution path of service-oriented manufacturing and supply chain. Therefore, it is necessary to combine the evolution path of serviceoriented manufacturing supply chain network with the environment of enterprises. Based on the integration of internal and external environment, the mechanism of serviceoriented manufacturing evolution can be analyzed, and the specific path of the evolution process of service-oriented manufacturing supply chain can be described, which can point out the direction of enterprise transformation.

\section{CONCLUSION}

By studying the evolutionary path of service-oriented manufacturing supply chain, people can more fully understand the driving force and feasibility of the transformation from traditional manufacturing to serviceoriented manufacturing. Also, it can help the enterprises accelerate the transformation of enterprises, make up for the deficiencies of the current research on supply chain network of service-oriented manufacturing, and provide theoretical basis for subsequent research. The results of research on evolution path of supply chain network in service-oriented manufacturing industry can be used as a theoretical reference for the transformation of China's manufacturing industry, such as electronic manufacturing industry, household appliances manufacturing industry and other industries to service-oriented manufacturing industry, and for the construction of supply chain network in service-oriented manufacturing industry, so as to speed up the realization of the national industry-wide intelligent supply chain system and promote the realization of China's 2025 strategic plan of manufacturing powerful nation.

The service-oriented manufacturing supply chain is more complex than the manufacturing supply chain network and the service supply chain network. It is necessary to study the evolution path of the service-oriented manufacturing supply chain network from the following aspects:

- To clarify the impact of environmental factors on the transition from traditional manufacturing to serviceoriented manufacturing;

- To define the connotation of service-oriented manufacturing supply chain and the driving force of its evolution correctly;

- To construct the supply chain network structure of service-oriented manufacturing industry, and construct its evolutionary mathematical model; 
- To use simulation software to simulate the evolution path of supply chain network in service-oriented manufacturing industry, so as to truly reflect the evolution process.

\section{REFERENCES}

[1] Timothy D. Fry,Daniel C. Steele, Brooke A. Saladin. A Serviceoriented Manufacturing Strategy [J]. International Journal of Operations \& Production Management . 1994-10

[2] Fujimoto Jun, Umeda Yasushi, Tamura Tetsuya,Tomiyama Tetsuo. Kimura Fumihiko; Development of service-oriented products based on the inverse manufacturing concept $[\mathrm{J}]$, Environmental Science \& Technology, 2004-23

[3] Sun Linyan, Li Gang, Jiang Zhibin, Zheng Li, He Zhe. Advanced Manufacturing Model in the 21st Century - Service Manufacturing [J], China Mechanical Engineering. 2007-10. (in Chinese)

[4] Rubén Darío Franco,Ángel Ortiz Bas,Francisco Lario Esteban. Modeling extended manufacturing processes with service-oriented entities [J]. Service Business.2009-1

[5] Jiaqi Yan, Kang Ye, Huaiqing Wang, Zhongsheng Hua.Ontology of collaborative manufacturing: Alignment of service-oriented framework with service-dominant logic [J], Expert Systems With Applications. 2009-3

[6] Xi Zhuyan, Zheng Bo, Sun Linyan. Service-oriented Manufacturing Strategy: Solving the Lag of Service Industry and Manufacturing Industry in Shandong Province [J], Journal of Shandong University (Philosophy and Social Sciences Edition). 2009-01. (in Chinese)

[7] LIN Wenjin, Jiang Zhibin, Li Na. A Survey on the Research of Service-Oriented Manufacturing [J], Industrial Engineering and Management. 2009-12. (in Chinese)

[8] Hasse Nylund,Paul H. Andersson.Simulation of service-oriented and distributed manufacturing systems [J], Robotics and Computer Integrated Manufacturing.2010-6

[9] Paulo Leitão,Joel Alves,Ana I. Pereira.Solving Myopia in Real-time Decision-making using Petri nets Models' Knowledge for Serviceoriented Manufacturing Systems [J], IFAC Proceedings Volumes. 2010-4

[10] Wang Mingwei, Zhang Shusheng, Zhou Jingtao. Framework of collaborative business processes in service-embedded manufacturing paradigms [J]. Computer Integrated Manufacturing Systems. 2010-11. (in Chinese)

[11] Shuangxi Huang,Sen Zeng,Yushun Fan,George Q.Huang. Optimal service selection and composition for service-oriented manufacturing network $[\mathrm{J}]$. International Journal of Computer Integrated Manufacturing.2011-5

[12] Jie Gao,Yinliang Yao,Valerie C. Y. Zhu, Linyan Sun,Lin Lin. Service-oriented manufacturing: a new product pattern and manufacturing paradigm $[\mathrm{J}]$. Journal of Intelligent Manufacturing. 2011-3

[13] Cheng Gongsheng. Guangdong province developing service style manufacture industry's facing problem and countermeasure research [J], Special Zone Economy, 2011-03. (in Chinese)

[14] Chen Juhong, Jiao Xingjia, Guo Fuli. Design of product service system under service-oriented manufacturing [J], Statistics and Decision. 2011-07. (in Chinese)

[15] Cui Jiachen, Lin Wenjin, Wang Shuai and Jiang Zhibin. Research on the Customer Value Delivery Mechanism in Service Manufacturing [J], Industrial Engineering and Management. 2011-08. (in Chinese)

[16] Corina Popescu,M. Cavia Soto,Jose L,Martinez Lastra. A Petri netbased approach to incremental modelling of flow and resources in service-oriented manufacturing systems [J], International Journal of Production Research.2012-2

[17] H. Guo,F. Tao,L. Zhang,Y. J. Laili,D. K. Liu.Research on measurement method of resource service composition flexibility in service-oriented manufacturing system[J], International Journal of Computer Integrated Manufacturing.2012-2
[18] Fei Tao,Ying Cheng,Lin Zhang,Dongming Zhao.Utility modelling, equilibrium, and coordination of resource service transaction in service-oriented manufacturing system $[\mathrm{J}]$, Proceedings of the Institution of Mechanical Engineers.2012-6

[19] Fuqiang Zhang, Pingyu Jiang, Qiqi Zhu, Wei Cao. Modeling and analyzing of an enterprise collaboration network supported by service-oriented manufacturing [J], Proceedings of the Institution of Mechanical Engineers.2012-9

[20] Lu Zhen.An analytical study on service-oriented manufacturing strategies [J]. International Journal of Production Economics.2012-1

[21] Cheng Dongquan, Gu Feng, Geng Yong. Research on the construction and operation Mechanism of Value Chain System in Service-oriented Manufacturing [J], Management World, 2011-12. (in Chinese)

[22] Yao Qifu, Xiong Huiping, Wang Hongjun. Research on the Implementation Path of Service-oriented Manufacturing: Taking Ningbo Manufacturing Industry as an Example [J], Enterprise Economy. 2012-05. (in Chinese)

[23] Xie Wenming, Jiang Zhibin, Chu Yanbing. Application of Service Oriented Manufacturing in Traditional Manufacturing.a Case Study of Shanghai Electric [J], Industrial Engineering and Management, 2012-12. (in Chinese)

[24] Cristina Morariu,Octavian Morariu,Theodor Borangiu.Formalized Information Representation for Intelligent Products in ServiceOriented Manufacturing [J], Yves Sallez IFAC Proceedings Volumes. 2013-7

[25] Yang Lixi, Tang Zhenpeng. Development Thought and Countermeasure of Service-oriented Manufacturing Model in Haixi Economic Zone [J], Southeast Academic Research. 2013-09. (in Chinese)

[26] Kangzhou Wang, Zhibin Jiang, Na Li, Na Geng.Optimal production control of a service-oriented manufacturing system with customer balking behavior [J]. Flexible Services and Manufacturing Journal. 2014-3

[27] Yang Hui, Song Huaming, Yu Anping. Theoretical Analysis and Empirical Study on Competitive Advantages of Service-oriented Manufacturing-Based on the Data of 200 Manufacturers in Jiangsu Province [J], Management Review. 2014-03. (in Chinese)

[28] Bai Li Guo.The Research of Supply Chain Optimization Theory Based on Service-Oriented Manufacturing [J]. Applied Mechanics and Materials.2015-733

[29] Zhang Zhong, Jin Qing. Research on Manufacturing Industry Value Creation based on Service-oriented Manufacturing Network [J]. Commercial Research, 2015-04. (in Chinese)

[30] Jin Cao, Zhibin Jiang, Kangzhou Wang. Customer demand prediction of service-oriented manufacturing incorporating customer satisfaction [J]. International Journal of Production Research. 2016-5

[31] Liangqing Feng,Kunkun Zeng,Fangling Shu.Study on the Quality Control of Reputation-Based on Incentive Service-Oriented Manufacturing Network [J]. Open Journal of Social Sciences.2016-07

[32] Liu Zhiyong, Li Bizhen, Ye Songzhong, Yang Shaoxiong. Serviceoriented Manufacturing: Research on the Supply-side Reform Path of Fujian Sports Goods Manufacturing Industry [J], Journal of Fujian Normal University(Philosophy and Social Sciences Edition). 2016-09. (in Chinese)

[33] Ying Cheng, Fei Tao, Dongming Zhao, Lin Zhang. Modeling of manufacturing service supply-demand matching hypernetwork in service-oriented manufacturing systems [J]. Robotics and Computer Integrated Manufacturing.2017-10

[34] Jian Zhaoquan, Liu Xiaoyan, Li Lei. "Platform + Small and Micro Enterprises" Organization Structure for Service-Oriented Manufacturing Enterprises - A Case Study of Haier [J], Chinese Journal of Management. 2018-11. (in Chinese)

[35] Julia Siderska,Khair S. Cloud manufacturing: a service-oriented manufacturing paradigm.A review paper $[\mathrm{J}]$. Jadaan Engineering Management in Production and Services.2018-1

[36] Yan Kaining, Li Gang. Enterprise Evolution of Service-oriented Manufacturing under the Context of "Internet Plus" [J]. China Mechanical Engineering. 2018-09. (in Chinese) 
[37] David Sadler. Cluster Evolution, the Transformation of Old Industrial Regions and the Steel Industry Supply Chain in North East England [J]. Regional Studies 2004-1

[38] Li Jizi, Cai Gennv, Lu Deyin. Research on the Evolution Law of Cluster Network Supply Chain Change [J]. Journal of Intelligence. 2004-02. (in Chinese)

[39] Wang Yongping, Meng Weidong. Evolutionary Game Analysis on Co-competition Mechanism of Supply Chain [J], Journal of Industrial Engineering and Engineering Management. 2004-06. (in Chinese)

[40] Masayoshi Maruyama,Mitsunori Hirogaki.The evolution of fresh produce supply chains: From spot markets to contracts [J]. The International Review of Retail, Distribution and Consumer Research 2007-4.

[41] Wang Ling. Co-opetition Mechanism in Supply Chain Network: An Evolutionary Game Theory Approach [J], Forecasting. 2007-09. (in Chinese)

[42] Tim Coltman, Kathrine Bru, Nidthida Perm-Ajchariyawong, Timothy M.Devinney. Supply chain contract evolution [J]. Gabriel R.G. Benito European Management Journal 2008-6

[43] V. Daniel R. Guide,Luk N. Van Wassenhove .OR FORUM-The Evolution of Closed-Loop Supply Chain Research [J]. Operations Research 2009-1

[44] Wang Shilei, Yan Guangle. Evolutionary model between suppliers and core-enterprise in green supply chains [J], Science-Technology and Management. 2009-05. (in Chinese)

[45] Ozcan, Ozan, Reeves, Kingsley, Ozcan,Deniz.Supply Chain Structure Evolution of Sustainability-Focused Companies (Presentation) [J]. IIE Annual Conference Proceedings .2010.PAGES:1-22

[46] Chai Guorong, Li Zhenchao, Wang Xiaogeng, Zong Shengliang. Evolutionary game analysis on the cooperative behavior in industrial cluster under supply chain networks [J], Science Research Management. 2011-05. (in Chinese)

[47] Hou He, Shouyang Wang, T.C.E. Cheng. Competition and evolution in multi-product supply chains: An agent-based retailer model [J]. International Journal of Production Economics 2013-1

[48] Ji,Guojun, Gunasekaran, Angappa. Evolution of innovation and its strategies: from ecological niche models of supply chain clusters [J]. The Journal of the Operational Research Society .2014-6

[49] Wu Yisheng. Evolution Model of Low Carbon Supply Chain Collaborative Operation [J], Operations Research and Management Science. 2014-04. (in Chinese)

[50] Xiao Xue, Shufang Wang, Hao Chao. Autonomous evolution of service system in cluster supply chain [J]. Kybernetes 2015-1

[51] Zhang Guoxing, Fang Shuai, Wang Yingluo. Analysis of Supply Chain Coordination Mechanism Based on Evolutionary Game [J], Statistics and Decision. 2015-08. (in Chinese)

[52] Ying $\mathrm{Xu}$, Xuemei Zhang, Jian Cao, Ya Chen, Xuhong Ye, Paolo Renna. Collaboration and Evolution of E-Commerce and Express Delivery Industry Supply Chain [J], Discrete Dynamics in Nature and Society .2016- 10 .

[53] Wang Ying, Zhang Chun. Research on Supply Chain Governance Pattern and Evolutionary Process [J], China Business and Market. 2017-02. (in Chinese)

[54] Hua Wang, Tao Gu, Maozhu Jin, Rong Zhao, Guanxiang Wang Chaos. The complexity measurement and evolution analysis of supply chain network under disruption risks $[\mathrm{J}]$, the interdisciplinary journal of Nonlinear Science. 2018-10

[55] Tang Run, Peng Yangyang. Analysis of dual-Channel Coordination Evolutionary Game in Fresh Food Supply Chain Considering Channel Characteristics [J], Statistics and Decision. 2018-07. (in Chinese)

[56] He Binbin, Liu Qin. Analysis on Evolutionary Game of Vertical Cooperation in Supply Chain Considering Consumer's Green Preference [J], Statistics and Decision. 2018-07. (in Chinese)

[57] Yunus Kathawala, Khaled Abdou.Supply chain evaluation in the service industry: a framework development compared to manufacturing [J]. Managerial Auditing Journal. 2003-2
[58] Kaushik Sengupta,Daniel R. Heiser,Lori S. Cook.Manufacturing and Service Supply Chain Performance: A Comparative Analysis [J]. Journal of Supply Chain Management .2006-4

[59] Wang Chunzhi, Heiko Gebauer, Sun Liyang. Build up of Service Management Strategy System of Manufacturing Company Based on Supply Chain Management [J], Journal of Changchun University of Science and Technology. 2008-01. (in Chinese)

[60] He Zhe, Sun Linyan, He Zhuqing, Li Gang. Trend of Servicemanufacturing and Differences between Service- manufacturing Network and SCM [J]. Soft Science. 2008-04. (in Chinese)

[61] Wang Jipeng, Xiong Jing, Wang Tianlin. Interface Implementation of Manufacturing Industry Agile Supply Chain Nodes Based on Service Agent [J]. Procedia Environmental Sciences. 2011-1

[62] Tang Yan, LI Jian, ZHANG Ji-hui. Cloud manufacturing service platform design of closed-loop supply chain oriented to remanufacturing $[\mathrm{J}]$. Computer Integrated Manufacturing Systems. 2012-07. (in Chinese)

[63] Wang Kangzhou, Jiang Zhibin, Lin Wenjin, Xie Wenming. Study on the hybrid supply chain management of service-oriented manufacturing [J], Soft Science. 2013-05. (in Chinese)

[64] Ma Chao, Wang Jun, Gao Song. Evaluation of Coordination Capability of Service-oriented Manufacturing Hybrid Supply Chain [J], Commercial Time. 2013-12. (in Chinese)

[65] Jukka Hemilä, Jyri Vilko. The development of a service supply chain model for a manufacturing SME [J], The International Journal of Logistics Management. 2015-3

[66] James Aitken, Paul Childerhouse, Eric Deakins, Denis. A comparative study of manufacturing and service sector supply chain integration via the uncertainty circle model [J], Towill The International Journal of Logistics Management. 2016-1

[67] Ray Y. Zhong, Stephen T. Newman, George Q. Huang, Shulin Lan. Big Data for supply chain management in the service and manufacturing sectors: Challenges,opportunities, and future perspectives [J], Computers \& Industrial Engineering. 2016-10

[68] Dan Bin, Luo Xiao, Liu Molin. Product service supply chain modes based on process integration of manufacturing and service. Journal of Chongqing University (Social Sciences Edition). 2016-01. (in Chinese)

[69] Wang Weiwei, Hong Yue, Zhang Zaifang. Assessing stability and resilience of service-manufacturing hybrid supply chain [J], Computer Integrated Manufacturing Systems. 2017-07. (in Chinese)

[70] Ehsan Pourjavad, Arash Shahin. Hybrid performance evaluation of sustainable service and manufacturing supply chain management: An integrated approach of fuzzy dematel and fuzzy inference system [J], Intelligent Systems in Accounting, Finance and Management. 2018 07

[71] Cao Yuping. Approach Analysis of Developing Service-oriented Manufacturing in Textile Industry Transformation Area - Based on the Case of Weifang. China Textile Leader. 2017-07. (in Chinese) 